\title{
LOS COLORES DEL PARDO EN EL PARAGUAY A PARTIR DE DATOS DE FILIACIÓN DE FUGADOS Y DESERTORES ENTRE 1862 Y 1865
}

GARCÍA RIART, Jorge ${ }^{1}$

https://orcid.org/0000-0001-9728-3702

RESUMEN: A partir de los datos de filiación de 7 casos de soldados y reos desertores y fugados entre 1862 y 1865 extraídos de la Sección Historia del Archivo Nacional de Asunción se construye una métrica experimental de tonos de la tez atribuidos a los pardos en el Paraguay. La colorimetría puede ser una técnica para poner a prueba la hipótesis del oscurecimiento de la tez del paraguayo y la paraguaya contrario a la tradición nacionalista que sostiene cierta originalidad en el proceso de conformación del mestizo o del criollo paraguayo desprendido de la mezcla con el negro. A pesar de la graduación de colores, el adjetivo pardo, no solo como color de piel, permanece transversal como una casta social como resultado de la mezcla de un blanco con negra o viceversa y también de un negro con india o viceversa

PALABRAS CLAVE: Pardo; mestizo; criollo; mulato; colorimetría.

\begin{abstract}
From the filiation data of 7 cases of soldiers and inmates deserters and escaped between 1862 and 1865 extracted from the History Section of the National Archive of Asunción, an experimental metric of complexion tones attributed to Pardos (brownmen) in Paraguay is constructed. Colorimetry can be a technique to test the hypothesis of the darkening of the Paraguayan and Paraguayan complexions contrary to the nationalist tradition that maintains certain originality in the process of shaping the mestizo or the Paraguayan creole detached from the mixture with black. Despite the graduation of colors, the adjective Pardo (brownman), not only as a skin color, remains transversal as a social caste as a result of the mixture of a Whiteman with a Blackman or vice versa and also a Blackman with American Indian.
\end{abstract}

KEYWORDS: Pardo (brownman); mestizo; creole; mulatto; colorimetry.

1 Director Académico del Centro de Políticas Públicas de la Universidad Católica N.S.A., presidente del Centro de Investigaciones de Historia Social del Paraguay y miembro del Consejo del Centro de Estudios Antropológicos de la Universidad Católica. E-mail: jordiriart@yahoo.com 


\section{INTRODUCCIÓN}

Para la historia social del Paraguay, es importante recuperar indicios no hegemónicos desprendidos de una producción historiográfica paraguaya predominante en el siglo XX. Con intenciones ideológicas se formó un corpus teórico que contribuyó a crear una conciencia nacional despegada de factores comunes en la Región como la mestización de los negros.

Por ejemplo de esta situación, explícitamente, los negros no aparecen en los libros de historia, mientras tanto, la apología a la raza paraguaya como original prevaleció en la literatura local. Muchos autores, aun los más exquisitos, cometieron este desliz.

Este sistemático modo de narrar la historia olvidó a protagonistas civiles y resaltó, en contraposición, los valores de los héroes militares. Así también pasó con los negros introducidos al Paraguay, aunque con más dureza, fueron borrados literalmente del proceso de formación de la nación.

Rosana Güber dice que la etnografía como enfoque no pretende reproducirse según paradigmas establecidos, sino vincular teoría e investigación favoreciendo nuevos descubrimientos (GÜBER, 2001, p. 20). Nuestra intención, por tanto, es rescatar de la ausencia a otros actores sociales de la historia del Paraguay, como son los negros. Pero, ellos ya no están presentes y eso dificulta el estudio etnográfico basado exclusivamente en la observación.

Para salvar el problema metodológico, nos valemos de fuentes primarias conservadas en el Archivo Nacional de Asunción (ANA), principalmente, en la Sección Historia. De ahí, seleccionamos 7 casos de soldados desertores y fugados catalogados en notas de filiación (conjunto de datos personales) como pardos, sea este el apelativo a un color o un estatus social ${ }^{1}$.

La hipótesis es que los blancos en el Paraguay se ennegrecieron mediante la mezcla con negros, aunque es probable que también éstos últimos se hayan emparejado con indígenas. La técnica de la colorimetría puede ser una técnica práctica para entender ese probable proceso de obscurecimiento de la piel del paraguayo y la paraguaya.

Para que la exposición sea clara, nos apropiamos de la recomendación de David Jacobson con respecto a los elementos del texto etnográfico. Primero planteamos la pregunta o el problema, luego hacemos la explicación o la interpretación de la situación planteada, de aquí pasamos al ordenamiento de los datos donde se incluyen las evidencias documentales y finalmente llegamos a la conclusión como

$1 \quad$ Aclaración. No colocamos entre comillas los distintos apelativos de la colorimetría para evitar aludir a otros sentidos connotativos, a no ser que sean extractos literales de los autores. 
"en una secuencia argumental" -como dice el autor- (JACOBSON, 1991, p. 2).

\section{PROBLEMA: RECONOCER LA NEGRITUD}

¿De qué color era el pardo en el Paraguay? La respuesta puede ser obvia. Pero en la historiografía paraguaya clásica hay rotundos espacios blancos con relación a la negritud. El hombre y la mujer negros fueron borrados de la formación identitaria nacional.

"En ninguna colonia latina había tanta población blanca como en el Paraguay", se ufanó en explicar Manuel Domínguez en su célebre El Alma de la Raza (DOMíNGUEZ, 1917, p. 21). Justo Prieto, para justificar su Paraguay, la Provincia Gigante de las Indias, también subrayó que "a diferencia de lo que corresponde considerar en la mayoría de los países americanos, de la población paraguaya debe excluirse casi por completo al negro" (PRIETO, 1951, p. 52). Por su parte, Justo Pastor Benítez reprodujo en su Formación Social del Pueblo Paraguayo una colaboración de Carlos Gatti que sostenía que el paraguayo tomó del español "parte el cutis blanco" (BENÍTEZ, 1955, p. 90).

Si se sostiene que no hubo negros en el Paraguay asociado a la formación de la identidad nacional por qué mayoritariamente el paraguayo y la paraguaya son morenos. Jorge Rubiani en sus Historias secretas del Paraguay pregunta acaso "¿eran morenos los indígenas del Paraguay?" (RUBIANI, 2014, p. 70).

Carlos Gatti (léase en Benítez, 1995:90) señala que la fisonomía del guaraní pasó un proceso inverso: se blanqueó por la mezcla con el español, así la raza paraguaya "se asemeja a la europea como los observadores han admitido siempre que era una raza blanca pura".

Entonces, ¿cómo fue posible este melange de colores?, pregunta Alfredo Boccia Romañach. Una explicación posible, en el caso paraguayo, es que la mayoría de las esclavas negras tuvieron vástagos de color blanco, por lo cual la pureza de la raza negra no se mantuvo como sucedió en otros lados (BOCCIA ROMAÑACH, 2004, p. 253).

Otra tesis que perduró como argumento para emblanquecer la presencia del negro en el Paraguay tiene relación con cierto trato diferenciado con respecto a otros países esclavistas. J. R. Rennger sostuvo, según vio entre 1818 y 1826 , que la esclavitud en el país era mucho mejor que en otras latitudes (RENNGER, 2010, p. 103). John Hoyt Williams (WILLIAMS, 1995) propuso que no todos los afrodescendientes llegados al Paraguay fueron tratados como hermanos en alusión a la obra de Josefina Plà Hermano Negro, la esclavitud en el Paraguay (1972). Sin embargo, el aporte sustancial de Plà es reconocer que la mezcla de negros con la población local comenzó ya en la época de la Colonia, "aunque es verdad 
que esa mezcla no se redujo, por cierto, a la conjugación de la sangre blanca con la negra" (PLÀ, 2011:25).

Oscar Creydt minimizó también el papel de los negros en la Formación Histórica de la Nación Paraguaya. "La casta inferior de negros y mulatos tuvieron una importancia relativamente pequeña", señaló en su tesis (CREYDT, 2010, p. 64). Es decir, incluso el marxismo local se aferró a la idea de que el criollo mestizo (no mezcla de negros) como casta tuvo un estatus superior en el Paraguay. Pero Creydt hizo una interpretación económica, no social, con respecto a los negros.

La línea economicista que restó importancia a la influencia de los negros en el Paraguay continuó con Justo Pastor Benítez quien adujo que en el país no hubo grandes explotaciones mineras ni extensiones de cultivo para vincular a los esclavos negros en altas proporciones. El negro fue reducido a la servidumbre, "era más bien casera", explicó (BENÍTEZ, 1955, p. 81).

Antonio González, en un monográfico intitulado "El Paraguay, hombre americano", presentó a la sociedad paraguaya colonial dividida en 5 estamentos sociales: la capa superior o del español europeo, la capa del español americano, el criollo o mestizo (descendiente del conquistador), el indio libre o vasallo de la Corona, el indio guaraní mitayo y el indio guaraní yanacona, estas últimas clasificaciones de indígenas sujetos a encomiendas. Siguiendo esta corriente conservadora, agrega que a estas capas sociales, ya en los años 1700, se agregaron los de negros, mulatos y libertos en condición de inferioridad a los precedentes (GONZÁLEZ, 1969).

Por su lado Plá, explicó que en el Paraguay la mixtura genética entre negros, blancos e indígenas no representó grados nominativos como tercerón, cuarterón, quinterón, octavón, zambo o zambaigo, entre otros. Las epítetos más comunes a las personas no blancas fueron negro, pardo y mulato "con las consabidas imprecisiones" (PLÀ, 201, p. 27).

\section{MULATOS, MESTIZOS Y PARDOS EN EL PARAGUAY}

La presencia del negro no es eludible del Paraguay colonial ni tampoco del republicano. Los negros no pudieron pasar desapercibidos. Como dice Rubiani: "De hecho, no pasó inadvertida aunque fue tendenciosamente minimizada -cuando no tenazmente ignorada- por algunos de nuestros autores de textos escolares de historia" (RUBIANI, 2014, p. 71).

En la literatura local encontramos en cambio referencias al mestizo libre, que según Branislava Susnik, era aprobado generalmente por el Gobernador en sus visitas a 
los pueblos. Libre significa que estaba exento del trabajo obligado, de la vida comunal y de algún tributo específico. Así surgió el estatus socioracial de indio libre y de pardo libre (SUSNIK, 1965, p. 205).

En algún momento, se introdujeron en el Paraguay mulatos extranjeros. Jerry Cooney recogió un listado de nuevos residentes que fueron censados en 1804 por el gobernador Lázaro de Ribera. Entre los obreros que entraron a la Provincia figura un tal José de Acosta, mulato libre, soltero, 25 años (COONEY, 1990, p. 185). Es extraño que esta condición se haya grabado en el índice de registros de extranjeros. Generalmente, se excluían a las mujeres y a los niños inmigrantes. Tampoco -dice

Cooney- se tenía en cuenta a los negros nacidos en el extranjero, sean esclavos importados o prófugos del Brasil (COONEY, 1990, p. 194). Sin embargo, es un indicativo que seguía llegando gente no blanca a la Provincia en los primeros años del siglo XIX.

Según Plá, las palabras mulato o pardo fueron habituales en el Paraguay desde principio del siglo XVIII "cuando se habla de esclavos, lo mismo que cuando se habla de individuos libres, mestizos, en oposición a los términos moreno o negro, que designan al individuo de raza pura" (PLÀ, 2011, p. 27).

La prolífica periodista le da la razón a Félix Azara quien afirmó que el adjetivo pardo se utilizaba en la lengua para mencionar a toda persona afromestiza, sin importar su origen: descendiente de blanco y negro, de negro e indio o de blanco, negro e indio en las más diversas proporciones (PLÀ, 2011, p. 27).

Plà argumenta que poco el pigmento predominante pudo haber sido el blanco tanto en el Paraguay colonial como en el poscolonial. Hay pautas que los esclavos en la capital ofrecían un índice de mezcla de razas más elevado que en el interior, no sólo en las estancias y obrajes, sino en los mismos hogares de ciudades y pueblos (PLÀ, 2011, p. 22).

De cualquier modo, aunque complicado parezca saber la significación concreta de los colores atribuidos a las personas de tez obscura, que es el punto de nuestro interés, Ignacio Telesca plantea una hipótesis. Dice él que lo que se ha producido en el país más que un blanqueamiento de los negros es un ennegrecimiento de los blancos (TELESCA, 2008). De por sí polémica esta proposición, contradice la corriente nacionalista de fuerte vigencia en nuestro medio que configuró la nación paraguaya como un proceso de mestizaje muydistinto a lo producido en otros países de la Región.

\section{ALGUNOS RELATOS HISTÓRICOS}

El Dr. Johann Rudolf Rengger escribió que las mulatas jóvenes, en el Paraguay 
de Gaspar Rodríguez de Francia, cuando son blancas tienen más valor para los blancos que las desean como amantes (RENGGER, 2010, p. 79). ¿Mulatas blancas? ¿Qué sentido tenía este apelativo? El investigador da pistas para comprender la condición social de una persona mezclada con la discriminación racial. De hecho, el suizo reconoció que muchas veces la expresión fue empleada como un insulto.

El director de El Semanario, Ildefonso Bermejo, en la antesala de una audiencia con el presidente Carlos Antonio López, en 1855, contó que "salió a recibirnos un paraguayo de color algo más que trigueño" (BERMEJO, 1973:31). La expresión "algo más que trigueño" apuntaría a que el sirviente era más oscuro que un blanco. El publicista reconoció a muchos mulatos (literalmente en sus escritos) haciendo servicios domésticos o empleados en los servicios públicos en el Paraguay Lopizta. En un libro, contó esta situación: "El diálogo fue interrumpido por la llegada de un joven mulato, descalzo y en mangas de camisa, que nos anunció que el señor Presidente podía recibirme" (BERMEJO, 1973, p. 41).

Otros extranjeros que visitaron el Paraguay decimonónico recogieron en sus bitácoras el avistamiento de muchos negros. El norteamericano Thomas Jefferson Page, por ejemplo, escribió que "un negrito, que había estado durante la comida como una estatua detrás de las silla de su amo, de repente golpeó las manos y con la gravedad de un obispo, agradeció en reciprocidad con voz grave, muy peculiar" (PAGE, 2007:250-251). La descripción es tan elocuente, que en algunas ediciones del libro se incluye una litografía de esta escena. Hay más dibujos significativos sobre población negra en el país en las obras de Alfred Demersay y Charles Washburn (véase la compilación de Milda Rivarola, 2016, en la sección 6 "Lo peculiar de su gente").

Michael Mulhall, un editor irlandés, en su The cotton fields, dedicó un apartado a describir la influencia del indígena guaraní y su lengua en el mestizaje local: "La raza pura está ahora casi extinta”, escribió. En la obra se explica que había, en 1864, negros o mulatos aunque "muy pocos". "En Asunción, donde algunos de sangre blanca han quedado casi puros, el color de sus habitantes es más claro que los del interior"2 (MULHALL, 1864, p. 110).

“Angola, negra motuda ${ }^{3}$, piel de carbón”, así pintó Helio Vera a la protagonista principal de unos de sus cuentos más conocidos (VERA, 1984, p. 17), procurando significar la verdadera obscuridad de su piel. Angola, al parecer no fue una mujer de ficción; según

$2 \quad$ Traducción propia. La frase en castellano recuerda una interpretación de Josefina Pla mencionada en párrafos precedentes.

3 La expresión motuda alude a motas; se emplea para describir generalmente el pelo crespo o rizado. 
confesó una vez el escritor, fue una sirvienta de sus antepasados guaireños ${ }^{4}$. El nombre no es casual. De aquélla nación africana llegaron muchos esclavos y esclavas al Paraguay.

\section{COLORES DE PARTIDA}

Cualquiera haya sido la intención de negación del negro de la historia del Paraguay, aun cuando es explícita su presencia en los relatos históricos generalmente de extranjeros, no obstante, se puede reconocerse un proceso de mestizaje que moldeó ciertos grados nominativos y adjetivos tales como blanco, trigueño, mulato, negrito, "más claro", incluso el de raza pura.

Trigueño fue una apelativo corriente en el siglo XIX. Al respecto, El Nuevo Diccionario de la Lengua Castellana de Salvá (1846, p. 1976) hace referencia a "lo que tiene el color del trigo entre moreno y rubio" 5 . La misma fuente indica que negro6 ${ }^{6}$ significa que es "moreno o que le falta la blancura que le corresponde" (SALVÀ, 1846, p. 748).

Por su parte, negrito es una expresión de cariño usual (SALVÀ, 1846, p. 748), aunque alude indefectiblemente al color de la piel.

Las crónicas también traen vocablos en los que se entrecruzan el sentido del color y el sentido de la casta, tales como mulato o pardo. Según la consulta con el Diccionario de la época, mulato7 "se aplica a la persona que ha nacido de negra y blanco o al contrario"; agrega "lo que es de color moreno" (SALVÂ, 1846, p. 739). Por su lado, pardo", siguiendo la misma fuente, "se aplica al color que resulta de la mezcla del blanco y negro" (SALVA, 1846, p.801). Alegando la coincidencia que trae el Diccionario, podríamos decir que el color pardo es equivalente a mulato en la época de uso.

Debido a la repetición de moreno $^{9}$ en las entradas consultadas, nos valemos de nuevo del Diccionario de Salvá para aproximarnos al tono indicado. Dice que es aplicado generalmente al "hombre negro atezado por suavizar la voz negro, que es la que corresponde", aunque antepone al ejemplo que moreno "se aplica al color oscuro que tira a negro" (SALVA, 1846, p. 731). A su vez atezado, viene de tez, y para la comprensión

$4 \quad$ Nota del autor. No tengo registro concreto del testimonio expresado por el escritor Helio Vera, pero si la memoria del momento en que me transmitió ese detalle durante un viaje en automóvil en el que tuve el gusto de llevarlo a su casa.

5 Entrada: Trigueño, ña.

$6 \quad$ Entrada: Negro, gra.

$7 \quad$ Entrada: Mulato, ta.

8 Entrada: Pardo, da.

9 Entrada: Moreno, na. 
moderna sería bronceado o moreno ${ }^{10}$.

Si organizamos los colores aludidos mediante una métrica de graduación de tonos claros a tonos obscuros, podemos tener una matriz colorimétrica, poco habitual en las ciencias sociales pero útil para relacionar los adjetivos de colores (extraídos textualmente de las fuentes primarias) con los significados (según Diccionario) y los tonos; que ese es el punto que nos interesa. Entonces, puede ayudarnos a encontrar la respuesta al problema del color del pardo en el Paraguay.

En la Tabla 1, presentamos una matriz inicial de adjetivos de color, con sus respectivos significados y su asociación con tonos, para aproximarnos a una interpretación de las fuentes de filiación primarias de pardos que describiremos en adelante.

Tabla 1. Significado y tonos de colores en el Paraguay, siglo XIX

\begin{tabular}{ccc}
\hline Adjetivo & Significado & Tono \\
trigueño, ña & color del trigo & entre moreno y rubio \\
negro, gra & le falta blancura & moreno \\
pardo, da & mezcla del blanco y negro & moreno (por equivalencia) \\
mulato, ta & de negra y blanco, o viceversa & moreno \\
\hline
\end{tabular}

Elaboración propia a partir de SALVÀ, 1846

\section{ORGANIZACIÓN DE LOS CASOS}

El primer legajo del ANA que estudiamos se corresponde con la Sección Historia, volumen 342, número 6 y trata de una circular sobre desertores del Ejército en 1862 distribuido a varios jueces de paz, comisionados y jefes de urbanos del centro y sur del país principalmente. En realidad, se presenta un solo caso en la foja 1 y las demás fojas contienen recibos de los destinatarios de la orden que inquiría información sobre el paradero de un soldado desertor del Cuartel del 1er. Batallón de Asunción. El encabezado de la nota dice:

Los señores Jueces de Paz, Comisionados y Jefes de Urbanos de la ruta marginal se servirán inquirir con las mas vivas diligencias el paradero del soldado desertor Marcelino Godoy del partido de Capiata, el cual se desapareció de este Cuartel el dia 13 de diciembre de 1862, y habido que sea remitido bajo buena seguridad a disposición del infrascrito Coronel de Infantería y Mayor de Plaza de la Capital [Vicente Barrios]. (ANA, SH, vol. 342, n. 6, f. 1)

Marcelino Godoy tenía 30 años, 7 cuartas ${ }^{11}$ de altura (1,60 metros aproximadamente)

10 Real Academia Española: https://dle.rae.es/atezado

11 Una cuarta equivale aproximadamente a 22,86 centímetros; era una antigua forma de 
y era de color trigueño, según las señas aportadas por el informante. Otros rasgos descriptos son: pelo crespo, cejas grandes, ojos grandes, cara redonda, nariz corta y puntiaguda, boca grande, buena dentadura, labios gruesos, barba poca y cuerpo retacón.

En el mismo volumen documental, pero número 19, se encuentran los casos de dos soldados destinados a la obras de construcción del Ferrocarril en Pirayú y Paraguarí, respectivamente, quienes desertaron con una distancia de un año entre ellos. Uno se llamaba Francisco Vera (31 años) y otro Dionisio Escurra (41 años), ambos eran naturales de Villarrica.

Si bien la descripción de rasgos que aporta el informe con relación a los desertores dice que eran pardos libres, cuando escribe el color de la cara, incluye el adjetivo aindiado; esto quiere decir que tenían el color del indígena. Veremos más adelante qué tono representa el color señalado.

De Francisco, los datos de filiación grafican que tenía el cuerpo corpulento, estatura 1,52 metros, el pelo negro y liso, la cara redonda, los ojos chicos y negros, la nariz corta, la boca regular con buena dentadura y el bigote raso, sin barba. De Dionisio, señalaron las mismas señas con la única diferencia que carecía de dentadura.

El comandante afectado por la deserción de Vera, Francisco González, destinó a los puestos de vigía de la zona este rótulo:

Adjunto remito a U. la filiación no completa del soldado Francisco Vera del Batallón $\mathrm{n}$ 6 que fugó en este Campo, hoy [26/10/1862] á la lista de oración para que U. se sirva vigilar en el distrito en su mando hasta capturarlo y remitirlo á este Campo en la forma correspondiente. (ANA, SH, vol. 342, n.19, f. 2)

Y con relación a la fuga de Escurra, el comandante hizo el requerimiento obligado en estos términos:

Ayer día [3/09/1863] horas de la retreta fugó de este Campo un soldado perteneciente al Departamento de su Comando, cuya filiación adjunto a V.S. en copia legalizada, por todas las autoridades de su distrito, para la persecución y captura del relato desertor a fin de que reciba oportunamente este importante aviso, he tenido a bien enviarle propio a que ponga en sus manos la presente nota con la filiación misma. (ANA, $\mathrm{SH}$, vol. 342, n.19, f. 8)

Otros dos casos fueron recuperados de la Sección Historia, volumen 410, número 2, los cuales tienen relación con desertores de los cuadros de soldados destinados al Campamento de Sapucai donde también había empeño en terminar los rieles del Ferrocarril en 1864.

medir con la palma de la mano estirada. 
El oficial Francisco Toledo era oriundo de Santa María y estaba enrolado en la 1ª . Compañía, Batallón n. 7 del Ejército; soltero, 29 años, estatura 1,82 metros, cuerpo robusto, cara redonda, cabeza chica, pelo negro y crespo, cara redonda, picado por viruela, cejas poco pobladas, ojos en color sanguinolento, nariz delgada, boca regular con buena dentadura y lampiño, sin bigote. Su color: tostado. Francisco González agregó que tenía la espalda rota debido a castigos recibidos en una primera deserción.

Por su parte, el oficial Lázaro Barreto era natural de Limpio, soltero, 40 años, 1,60 metros de altura, cuerpo robusto, cabeza grande, pelo lizo, cara redonda, ojos negros, cejas poco pobladas, nariz chata, boca grande con buena dentadura y bigote bien poblado. Según la filiación, su color era moreno.

Los dos últimos casos de pardos fueron tomados de copias de circulares y decretos que se encuentran en la Sección Historia, volumen 345, número 11. Se tratan de Manuel Otazú y Fermín Acosta, quienes se escaparon de la carbonería de la Fábrica de Hierro de Ybycuí en 1865.

Los dos estaban presos; en el caso de Acosta, es explicito que estaba engrillado. El primero era vecino de Asunción y el segundo de Barrero Grande. En un solo folio se presentan los rasgos de los fugados: Manuel era de estatura alta (no especifica la medida), cuerpo delgado, pelo crespo, barba ralo (quiere decir poca); y Fermín era alto, algo fornido de cuerpo, cabello lacio y grueso y sin barba. El punto distintivo es que para el primero el color de piel se presenta como pardo mientras que para el segundo como tostado.

Transcribimos a continuación el folio completo donde se presentan los datos de filiación de Otazú y Acosta:

¡Viva la República del Paraguay!

El infrascrito $2^{\circ}$ Comandante de la Favrica de Fierro me dirijo a los Jefes de milicias y jueces de Paz de los partidos, de Quiquio, Mbuyapei, Caasapa, Yuti, Yhacaaguazu, Villa Rica, y sus departamentos Ytape, e Ybitimi, para que se sirvan las medidas mas convenientes á la Captura de dos presos nombrados Manuel Otazu y Fermin Acosta que han hecho fuga esta mañana del trabajo de la Carbonería de este Establecimiento; el primero vecino de la Capital, cuyas señas son: estatura alta cuerpo delgado color pardo pelo crespo pelo de Barba ralo y de su vestimenta lleva pantalón roto de casimir color café, camisa de hilo del coco, poncho criollo azul viejo, y sombrero de palma sucio, y el segundo con grillete vecino de Barrero grande, de estatura alto cuerpo algo fornido color tostado pelo liso y grueso y sin pelo de Barba y su Bestimenta que lleva, chiripa blanco de lana, camisa de hilo del coco, poncho caris ya viejo y sombrero de palma sucio [roto] se serviran remitir asegurados [roto]... (ANA, SH, vol. 345, n. 11, f. 10) 
Tabla 2. Casos de pardos fugados y desertores por año

\begin{tabular}{|c|c|c|c|c|c|c|c|}
\hline & Caso 1 & Caso 2 & Caso 3 & Caso 4 & Caso 5 & Caso 6 & Caso 7 \\
\hline $\begin{array}{l}\text { Año del } \\
\text { caso }\end{array}$ & 1862 & 1862 & 1863 & 1864 & 1864 & 1865 & 1865 \\
\hline Nombre & $\begin{array}{c}\text { Marcelino } \\
\text { Godoy }\end{array}$ & $\begin{array}{c}\text { Francisco } \\
\text { Vera }\end{array}$ & $\begin{array}{l}\text { Dionisio } \\
\text { Escurra }\end{array}$ & $\begin{array}{c}\text { Francisco } \\
\text { Toledo }\end{array}$ & $\begin{array}{l}\text { Lázaro } \\
\text { Barreto }\end{array}$ & $\begin{array}{l}\text { Manuel } \\
\text { Otazú }\end{array}$ & $\begin{array}{l}\text { Fermín } \\
\text { Acosta }\end{array}$ \\
\hline Ocupación & soldado & soldado & soldado & soldado & soldado & preso & preso \\
\hline Natural de & Capiatá & Villarrica & Villarrica & Santa María & Limpio & Asunción & $\begin{array}{l}\text { Barrero } \\
\text { Grande }\end{array}$ \\
\hline Edad & 30 años & 31 años & 41 años & 29 años & 40 años & sin dato & sin dato \\
\hline Estatura & $1,60 \mathrm{~m}$ & $1,52 \mathrm{~m}$ & $1,52 \mathrm{~m}$ & $1,82 \mathrm{~m}$ & $1,60 \mathrm{~m}$ & (alto) & (alto) \\
\hline Color & trigueño & aindiado & aindiado & tostado & moreno & pardo & tostado \\
\hline Situación & desertor & desertor & desertor & fugado & fugado & fugado & fugado \\
\hline Fuente & $\begin{array}{c}\text { ANA, SH, } \\
\text { vol. } 342, \text { n. } \\
6, \text { f. } 1\end{array}$ & $\begin{array}{l}\text { ANA, SH, } \\
\text { vol. } 342, \\
\text { n. } 19, \text { f. } 2\end{array}$ & $\begin{array}{l}\text { ANA, SH, } \\
\text { vol. } 342, \\
\text { n. } 19, \text { f. } 8\end{array}$ & $\begin{array}{c}\text { ANA, SH, } \\
\text { vol. } 410 \text {, } \\
\text { n. } 2 \text {, f.1 }\end{array}$ & $\begin{array}{l}\text { ANA, SH, } \\
\text { vol. } 410 \text {, } \\
\text { n.2, f.11 }\end{array}$ & $\begin{array}{l}\text { ANA, SH, } \\
\text { vol. } 345, \\
\text { n. } 11, \text { f.10 }\end{array}$ & $\begin{array}{l}\text { ANA, SH, } \\
\text { vol. } 345, \\
\text { n.11, f.10 }\end{array}$ \\
\hline
\end{tabular}

Elaboración propia

En la Tabla 2, presentamos un resumen de los casos de estudio en orden diacrónico, esto es por año de inscripción de la ficha de los casos documentales hallados en el ANA.

La organización de las fuentes nos permitió encontrar otros tonos de piel alusivos a los pardos que no pudimos hallar en relatos secundarios. La atención está puesta en los colores aindiado y tostado. El Diccionario de Salvá indica literalmente que aindiado"12 es "de color de indio, es decir entre rojo y cobrizo" (SALVÀ, 1846, p. 36). La voz parece provenir de Cuba. A su vez cobrizo 13 "es parecido al cobre en el color" (SALVÂ, 1846, p. 254). Tostado"14, según el Diccionario "dícese del color dorado subido y oscuro". La fuente explica que se llaman así "a varias castas de América" (SALVÀ, 1846, p. 1062). Pero, no obstante, debemos entender a cuál tono alude el dorado: "lo que es de color de oro" (SALVÀ, 1846, p. 417).

De esta manera, a la luz de los significados de las voces corrientes en la época de estudio para adjetivar al pardo, podemos completar la matriz de relaciones entre adjetivos, significados y tonos, como marca la Tabla 3.

\begin{tabular}{ll}
\hline 12 & Entrada: aindiado, da. \\
13 & Entrada: cobrizo, za. \\
14 & Entrada: tostado, da.
\end{tabular}


Tabla 3. Significado y tonos de colores de los pardos en el Paraguay, siglo XIX

\begin{tabular}{ccc}
\hline Adjetivo & Significado & Tono \\
\hline aindiado, da & color de indio & entre rojo y cobrizo \\
\hline trigueño, ña & color de trigo & entre moreno y rubio \\
\hline tostado, da & color dorado subido y oscuro & oro (oscuro) \\
\hline moreno, na & marrón oscuro & que tira a negro \\
pardo, da & mezcla del blanco y negro & moreno \\
\hline
\end{tabular}

Elaboración propia a partir de Salvà, 1846.

Ahora, a partir de una nueva graduación de tonos que se extiende entre claros e intensos, según la técnica de la colorimetría, podemos reagrupar los casos de pardos reos y fugados según la definición de los colores descriptos en las hojas de filiación. Véase Tabla 4.

Tabla 4. Ordenamiento de colores de pardos por tonos

\begin{tabular}{|c|c|c|c|c|c|c|c|}
\hline & Caso 7 & Caso 4 & Caso 2 & Caso 3 & Caso 1 & Caso 6 & Caso 5 \\
\hline $\begin{array}{l}\text { Color de } \\
\text { filiación }\end{array}$ & tostado & tostado & aindiado & aindiado & trigueño & pardo & moreno \\
\hline Tono & oro oscuro & oro oscuro & $\begin{array}{l}\text { e/ rojo y } \\
\text { cobrizo }\end{array}$ & $\begin{array}{l}\text { e/ rojo y } \\
\text { cobrizo }\end{array}$ & $\begin{array}{c}\text { e/ moreno } \\
\text { y rubio }\end{array}$ & moreno & $\begin{array}{l}\text { tira a } \\
\text { negro }\end{array}$ \\
\hline Fuente & $\begin{array}{l}\text { ANA, SH, } \\
\text { vol. } 345 \text {, } \\
\text { n.11, f.10 }\end{array}$ & $\begin{array}{c}\text { ANA, SH, } \\
\text { vol. } 410 \text {, } \\
\text { n. } 2, \text { f.1 }\end{array}$ & $\begin{array}{l}\text { ANA, SH, } \\
\text { vol. } 342 \text {, } \\
\text { n.19, f.2 }\end{array}$ & $\begin{array}{l}\text { ANA, SH, } \\
\text { vol. } 342 \text {, } \\
\text { n.19, f. } 8\end{array}$ & $\begin{array}{l}\text { ANA, SH, } \\
\text { vol. } 342, \text { n. } \\
6, \text { f. } 1\end{array}$ & $\begin{array}{l}\text { ANA, SH, } \\
\text { vol. } 345 \text {, } \\
\text { n. } 11, \text { f. } 10\end{array}$ & $\begin{array}{c}\text { ANA, SH, } \\
\text { vol. 410, } \\
\text { n.2, f.11 }\end{array}$ \\
\hline
\end{tabular}

Elaboración propia

En la nueva agrupación de casos, tenemos una configuración de la variedad de tonos que explican los colores de los pardos en el Paraguay. Hacia la derecha de la Tabla 4, los colores pardo y moreno se corresponden con tonos obscuros o casi negro (finalmente, pardo y moreno son sinónimos para la época de estudio como señalamos antes).

El tostado, por su alusión al tono dorado (por el oro) podría ubicarse entre los colores claros, aunque su sentido inicial es obscurecer la brillantez de algo. En definitiva, el aindiado y el trigueño pueden ser colocados como colores intermedios entre tonos claros y tonos obscuros. 
Tabla 5. Ordenamiento de colores de pardos por edad

\begin{tabular}{|c|c|c|c|c|c|c|c|}
\hline & Caso 7 & Caso 4 & Caso 1 & Caso 2 & Caso 5 & Caso 3 & Caso 6 \\
\hline Edad & $s / d$ & 29 años & 30 años & 31 años & 40 años & 41 años & $s / d$ \\
\hline Nacido en & $s / d$ & 1835 & 1832 & 1831 & 1824 & 1822 & $s / d$ \\
\hline Tono & oro oscuro & oro oscuro & $\begin{array}{l}\text { e/ moreno y } \\
\text { rubio }\end{array}$ & $\begin{array}{l}\text { e/ rojo y } \\
\text { cobrizo }\end{array}$ & tira a negro & $\begin{array}{l}\text { e/ rojo y } \\
\text { cobrizo }\end{array}$ & moreno \\
\hline Fuente & $\begin{array}{l}\text { ANA, SH, vol. } \\
345, \text { n.11 } \\
\text { f.10 }\end{array}$ & $\begin{array}{l}\text { ANA, SH, vol. } \\
410, \text { n.2, f.1 }\end{array}$ & $\begin{array}{l}\text { ANA, SH, vol. } \\
342, \text { n. } 6 \text {, f. } 1\end{array}$ & $\begin{array}{l}\text { ANA, SH, vol. } \\
342 \text {, n.19, f.2 }\end{array}$ & $\begin{array}{l}\text { ANA, SH, vol. } \\
410 \text {, n.2, f.11 }\end{array}$ & $\begin{array}{l}\text { ANA, SH, vol. } \\
342, \text { n.19, } \\
\text { f. } 8\end{array}$ & $\begin{array}{l}\text { ANA, SH, vol. } \\
345, \text { n.11, } \\
\text { f. } 10\end{array}$ \\
\hline
\end{tabular}

\section{Elaboración propia}

Otra posibilidad es ordenar los casos por años de nacimiento de los soldados desertores y fugados que seleccionamos, con ciertos errores ya que no pudimos confirmar todos los datos como se ve en la Tabla 5. Las fuentes para los casos 6 y 7 dicen explícitamente que están incompletos.

En esta nueva agrupación, se puede encontrar cierta evolución del tono con la edad. Los tonos claros coinciden con pardos jóvenes y los tonos obscuros con pardos viejos. Si el ejercicio fuera exacto, hasta podríamos colegir que el Caso 7 se trata de un pardo joven y el Caso 6 de un pardo más adulto. La impresión, en definitiva, es que los jóvenes son claros, y los viejos, obscuros.

\section{OTROS RASGOS}

Los traficantes de negros posiblemente hayan conservado registros en papel de los rasgos más representativos de sus víctimas relacionados con la edad, la salud, la estatura o la dentadura que se pasaban en medio de la compra y la venta. Algunas que se conocen tienen relación con el tiempo de arribo al mercado: negro bozal (recién llegado) o ladino (ya esclavizado en América). Otros apelativos identifican la estatura como negro de asta (por cumplir una determinada medida) así como la edad tal como muletón que eran los negros bozal de 10 a 15 años de edad (ARGÜELLO, 2012). Así, ciertas características descriptas parecen seguir un manual o guía preestablecidos.

Pero es muy probable que todos aquellos registros de filiación, que damos cuenta en este trabajo, hayan sido hechos desde la propia mirada de los funcionarios y ello puede comprometer la objetividad, aunque bien caracteriza la diversidad expresiva o de voces para 
representar un tono de piel.

Otros rasgos fenotípicos contribuyeron a la clasificación de los negros en complemento al color de piel. Susnik indica que en tiempos de la Colonia ciertas características del pelo, del cuerpo, de la cara, de la sangre constituyeron una fórmula habitual para conceder la condición social del negro (SUSNIK, 1965, p. 205). Argüello (2012) adiciona que la estatura de cada esclavo era valorada por una tablilla de palmos y los esclavistas hacían deducciones por aspectos físicos como la dentadura.

En los documentos analizados entre 1862 y 1865, encontramos interesantes citas de aquellos rasgos heredados de los papeles del mercado de la esclavitud aún cuando muchos de los casos comentados son nacidos ya después de la Colonia. Los casos se corresponden con nacidos entre 1822 y 1835, en época de José Gaspar Rodríguez de Francia, supremo dictador del Paraguay, cuando aún prevalecía la esclavitud porque los hijos de mujeres esclavas permanecían con tal condición a pesar de que sus progenitores sean blancos o indígenas. Además, los registros de filiación proceden de 10 años después de la abolición de la esclavitud por Carlos Antonio López, en 1853.

Sobre las características del rostro, los relatores (generalmente militares de alto rango) no escatiman adjetivos: grandes, redondas, cortas, entre otras. Sobre el cuerpo, emplean adjetivos tales como retacón, fornido, robusto, entre otros. Los detalles de la vestimenta coinciden con los uniformes de la soldadesca de la época.

Las descripciones de las características fenotípicas (cabello, nariz, boca, etc.), de vestimenta (ropa, calzado o descalzo, etc.), o de jerarquía militar (soldado, oficial) permiten ubicar a estas personas en la sociedad paraguaya desde su condición social.

Como sistematización de los datos de filiación apuntados a mano, elaboramos unas tablas para cada caso que están al final de este ensayo, ya que en estas hojas nos ocupamos solamente del color del rostro.

\section{CONCLUSIONES}

\section{Estratificación social}

Los casos presentados en estas hojas delatan la relación del pardo con el poder o con la élite dominante en la segunda mitad del siglo XIX. Describen situaciones de soldados y reos sometidos a tareas y servicios particularmente propios de la posición social que ocupaban en la sociedad.

La fuga y la deserción, sausas p̣or la que fueron buscados, explican la condición de sometimiento que padecieron las personas que hemos identificado, a partir de las señas de 
filiación que traen distintos colores como rasgos de identificación del pardo.

El tono de piel atribuido es consecuente con su estatus social disminuido frente a otras castas. Los indígenas estaban en condiciones superiores a los pardos. La graduación del color, en definitiva, representa una forma de cómo el lenguaje reproduce la dominación. Los voces moreno, pardo y trigueño, entre otros, tienen un significado de tono pero también un significado social, es atribución de la élite dominante sobre una casta inferior.

Esta interpretación aterriza actualmente con mucha fuerza en estudios de racismo y etnicidad en los Estados Unidos, con una denominación -quizás más apropiada a las ciencias sociales- que se conoce como colorismo (del inglés colorism). El concepto fue acuñado originalmente por Alice Walker en su libro In Search of Our Mother's Gardens: Womanist Prose. La autora considera que es una fuerza destructiva caracterizada por prejuicios y tratamientos preferentes de gente del mismo color basado solamente sobre su propio color (WALKER, 1983, p. 290). Hay estudios contemporáneos en África, Asia y América Latina que explican indicadores de ingreso, educación, incluso matrimonio como forma de discriminación social por el color de la piel (HUNTER, 2007). Por tanto, el colorismo no estudia las gamas de colores como a priori de la discriminación sino el nominativo de color de la piel a posteriori como estado de discriminación. Sobre Brasil, se están produciendo estudios historiográficos con relación al color del mestizaje como el trabajo de Viviane Weschenfelder y Mozart Linhares Da Silva (2018) quienes concluyen, a partir del análisis de la producción historiográfica de los siglos XIX y XX, que el pardo es la producción de subjetividades negras.

Aníbal Quijano (2014) plantea que la clasificación social ha sido un elemento constitutivo en la configuración del patrón mundial de poder, como piedra angular de la dominación del centro lo cual dio origen a nuevas identidades sociales tales como indios, negros, mestizos, entre otros o aceitunados, amarillos, blancos que evidencian un tono de color.

\section{Colorimetría del pardo}

La colorimetría ciertamente tiene que ver con la manipulación de la intensidad de los tonos puestos en una tabla según graduación. Como dijimos, está técnica no es atribuible generalmente a las ciencias sociales, empero contribuye, en este caso a intentar explicar los adjetivos aplicados a los pardos en el Paraguay decimonónico.

A partir de las definiciones de la época, pudimos construir una diversidad de tonos y encajar en ellas los adjetivos usados para señalar un rasgo de los pardos aludidos en los 
documentos de estudio.

Como pudimos entender, tras el rescate de las voces del Paraguay en el periodo de 1862 y 1865 , los significados derivados de los calificativos puestos a los pardos tienen relación generalmente con elementos de la naturaleza, sean estos oro, cobre, incluso trigo, por ejemplo.

Siguiendo el Gráfico 1, formado por esferas de colores, el adjetivo aindiado (rasgos de indio) transcurre entre la esfera de tono cobrizo y la esfera de tono rojo, siguiendo el significado del Diccionario de época. Por su parte, entre la esfera de tono moreno y la esfera de tono negro, se ubica el adjetivo mulato/pardo. El Diccionario dice-como anteriormente transcribimos- que es "una forma suave de decir negro". Por su lado, el adjetivo trigueño se entrecruza entre "rubio y moreno", como dice el Diccionario, lo cual puede ubicarse entre la esfera de tono oro y la esfera de tono bronce.

La colorimetría planteada en el gráfico no es exhaustiva ni tampoco acabada; su objetivo es delimitar el significado de los adjetivos de color aunque, cabe reconocer, que las fronteras muchas veces son difusas.

\section{Grafico 1. Colorimetría del pardo}

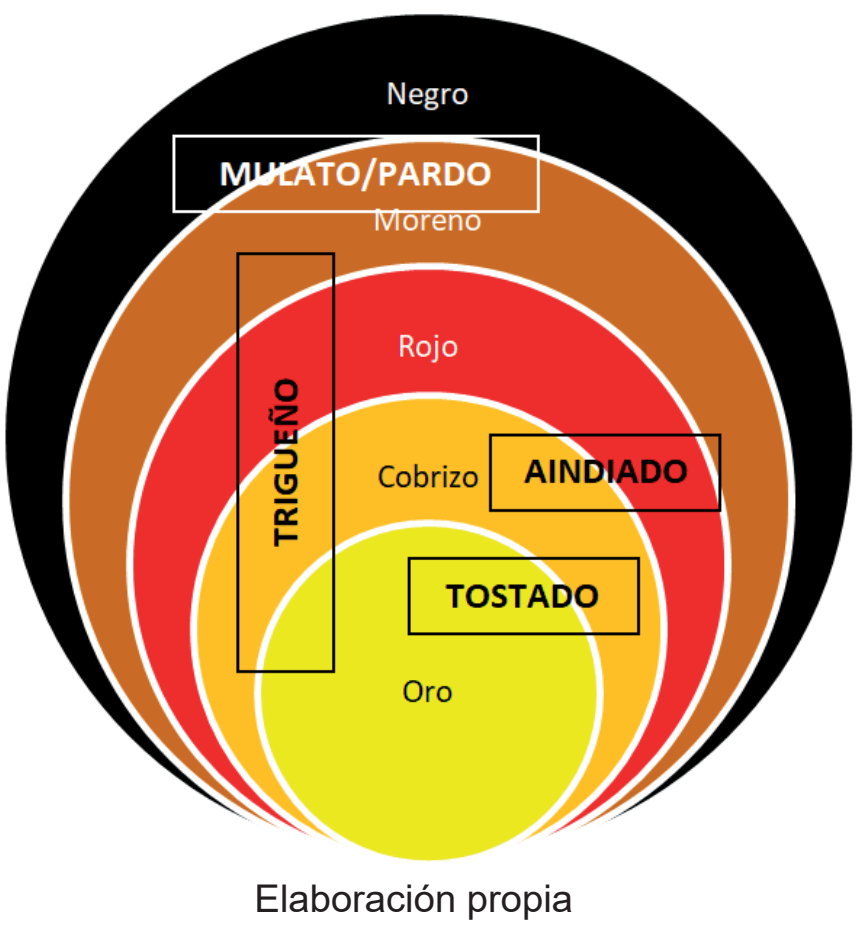

\section{Aindiado}

Si la acepción que trae el Diccionario de época con respecto a pardo tiene que ver con la mezcla de negro con blanca o viceversa, ¿qué significa color aindiado cuando 
identifican a un pardo? Básicamente, quiere decir que aindiado es atribuible a quien tiene los rasgos de indio en el color de la cara, en específico para dos casos que hemos encontrado en el ANA.

Eso quiere decir que el Diccionario no explica suficientemente la razón del pardo, que no solo es resultado de la unión de persona negra con persona blanca sino también con persona india, a no ser que el indio haya sido considerado intencionalmente como blanco.

Si comparamos los rasgos del pelo, según datos de filiación, tenemos que la persona identificada con el color pardo tiene el pelo crespo y la persona identificada con el color aindiado tiene el pelo liso, por citar dos características fenotípicas que distinguen a una persona de ascendencia africana y una persona autóctona de América, respectivamente.

Si aceptamos, a partir de estas evidencias, que la voz pardo como estatus social procede del adjetivo utilizado para mencionar a toda persona afromestiza, sin importar su origen: descendiente de blanco y negro, de negro e indio o de blanco, negro e indio, estamos coincidiendo con la observación de Azara que comentó Josefina Plá. ¿Pero también podríamos decir que los indígenas del Paraguay decimonónico eran morenos?

En definitiva, cabe la posibilidad que en el Paraguay se haya producido la mezcla de negros con indias o indios con negras y que esto haya resultado en una casta de pardos, seguramente de pardos libres pero no exentos de sometimientos y exclusiones.

\section{Pardo como casta}

Las evidencias documentales expuestas en estas hojas mostraron diferentes denominaciones de color con respecto al pardo, quizás por subjetividad de los funcionarios del Estado antes que por seguimiento de una forma o un registro objetivos.

Pero lo que permanece invariable en todos los casos analizados es el estatus como tal. Las fuentes primarias escogidas no hablan de mulato, aunque para el Diccionario consultado pardo y mulato serían lo mismo.

Estos hallazgos contradicen el empleo habitual del vocablo criollo o mestizo para referirse al paraguayo. Puede que este término sea más bien un simplismo para explicar la mezcla del indígena con el español blanco y no con la persona negra.

Las clasificaciones del color del pardo son consecuentes de su estatus social disminuida frente a otras castas. Los indígenas estaban en condiciones superiores a los pardos. Pero si los pardos son resultados de la fusión con indios ¿por qué permanece la discriminación social del pardo durante el siglo XIX?

Comprender los adjetivos de color utilizados en los datos de filiación para referir a 
una misma condición social, el pardo, es tarea que despierta la necesidad de profundizar más y mejor en la negritud del Paraguay.

La técnica experimental abre la posibilidad de practicar más búsquedas en las fuentes primarias para armar una matriz cultural de las castas sociales o mejor del negro en la sociedad paraguaya.

¿Qué pasó con los pardos?

Quedan abiertas algunas interrogantes para dar continuidad a estudios parecidos sobre la negritud en el Paraguay. Una de ellas tiene ver con el destino de los pardos del Paraguay. ¿Qué pasó con ellos? ¿Realmente perecieron en la Guerra contra la Triple Alianza - o como consecuencia de ella- o se convirtieron gradualmente dentro de un proceso de mestización que dio origen al fenotípico paraguayo?

La técnica de la graduación del color del pardo, ampliando cronológicamente la muestra y/o la cantidad de casos, puede contribuir a entender la hipótesis del ennegrecimiento de la sociedad paraguaya como un todo, comprobación o no a la que lógicamente deben ser agregados otros criterios sociales, económicos y políticos. ¿Es el paraguayo de hoy el pardo de ayer?

Al respecto, hay una imagen elocuente del estatus social de la gente morena o tostada en 1872 (Posguerra). En el diseño original del francés Édouard Riou a partir de un croquis de M. L. Forgues -reproducido muchas veces con distintos epígrafes en revistas y diarios extranjeros- aparece un grupo de más 25 personas (hombres, mujeres y niños en brazo) amontonadas en un vagón de tren al descubierto (en el original hay un vagón más abierto y otro cerrado que ocupan un par de hombres). La obra trae un importante detalle (véase Gráfico 2): las personas tienen todas rayadas en negro la piel a diferencia de la blancura de sus vestimentas. La cita textual del visitante dice explícitamente que aquel "vagón de pobres" estaba reservado para la gente negra ${ }^{15}$-"poor black folk", en el relato original- (HIELD, 1882, p. 187).

$15 \quad$ Traducción propia. 


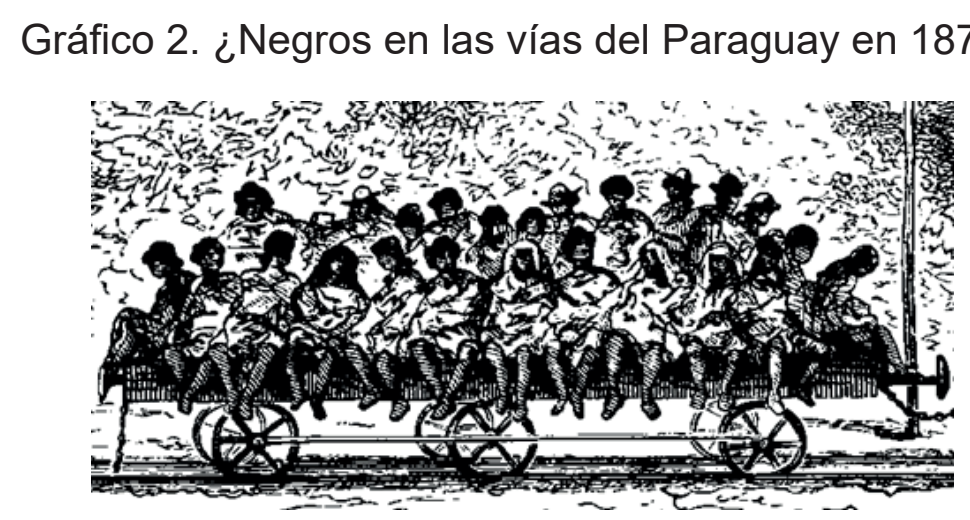

Fuente: Forgues, M. L. (1874).

\section{FUENTES}

\section{Archivo Nacional de Asunción (ANA)}

Sección Historia (SH), vol. 342, n. 6. Circular sobre desertores del Ejército, 1862

Sección Historia (SH), vol. 342, n. 19. Legajo de comunicaciones expedidas por el encargado de las obras del ferrocarril de Pirayú, 1862.

Sección Historia (SH), vol. 345, n. 11. Copia de circulares y decretos, 1865

Sección Historia (SH), vol. 410, n. 2. Correspondencias del pueblo de Sapucai, 1864.

\section{REFERENCIAS}

ARGUELLO, Ana María. Los negros en el Paraguay. Investigación especial 6. En RUBIANI, Jorge (Coord.). La historia del Paraguay, segunda edición. Asunción: Abc color, 2012, p.113128.

BERMEJO, Ildefonso. Vida paraguaya en tiempos del viejo López. Buenos Aires. Universitaria, 1973.

BOCCIA ROMAÑACH, Alfredo. Esclavitud en el Paraguay. Vida cotidiana del esclavo en las Indias Meridionales. Asunción: Servilibro, 2004.

COONEY, Jerry. Economía y Sociedad en la Intendencia del Paraguay. Asunción: CPES, 1990.

CREYDT, Oscar. Formación Histórica de la Nación Paraguaya. Asunción: Servilibro, 2010

DOMíNGUEZ, Manuel. El alma de la raza. Buenos Aires: Ayacucho, 1917.

FORGUES, M. L. Le Paraguay. Fragments de journal et des correspondances 1872/1873. En Le Tour du Monde. París: Hachette, 1er. semestre, 1874.

GARCÍA RIART, Jorge. Procesos a Postillones. Tres causas "criminales" en plena Guerra Grande, en Revista Paraguaya de Historia, vol. III, n. 1, julio 2020, pp. 67-81.

GONZÁLEZ, Antonio. El Paraguayo, un hombre americano. Guarania, Revista Paraguaya de Cultura, abril-junio, n. 2, p. 71-95, 1969,

HIELD CASSELL, Mary. Glimpses of South America or the land of the pampas. Londres: 
Gulpin \& Co., 1882.

HUNTER, M. The Persistent Problem of Colorism: Skin Tone, Status, and Inequality. Sociology Compass, 2007. p. 237-254.

JACOBSON, David. Reading Ethnography. Bufallo: Suny Press, 1991.

MULHALL, Michael George.The cotton fields. Paraguay and Corrientes... Buenos Aires, 1864.

PAGE, Thomas Jefferson. El Rio de la Plata, la Confederación Argentina y el Paraguay. Asunción. Intercontinental, 2007.

PLÀ, Josefina. Hermano negro: la esclavitud en el Paraguay. Madrid, 1972.

PLÀ, Josefina. La esclavitud en el Paraguay. Asunción. Intercontinental, 2011.

PRIETO, Justo. Paraguay. La Provincia Gigante de las Indias. Análisis espectral de una pequeña nación mediterránea. Buenos Aires: El Ateneo, 1951.

QUIJANO, Aníbal. Colonialidad y Modernidad/ Racionalidad, Perú Indígena, vol. 13, n. 29, p. 11-20, 1992,

RENGGER, L. R. Viaje al Paraguay en los años 1818 a 1826. Asunción: Tiempos de Historia, 2010.

RIVAROLA, Milda. Paraguay llustrado. Asunción: Servilibro, 2016

RUBIANI, Jorge. Historias secretas del Paraguay. Tomo II, Libro 4. Asunción: ABC, 2014.

SALVÁ, Vicente. Nuevo diccionario de la lengua castellana que comprende la última edición íntegra, muy rectificada y mejorada, del publicado por La Academia Española ... Paris, 1846.

SUSNIK, Branislava. El indio colonial del Paraguay. El Guaraní Colonial I. Asunción: Museo Etnográfico "Andrés Barbero", 1965.

TELESCA, Ignacio. La historiografía paraguaya y los afrodescendientes. En LECHINI, Gladys (Comp.). Los estudios afroamericanos y africanos en América Latina, herencia, presencia y visiones del otro. Clacso, 2008, p. 165-186.

VERA, Helio. Angola y otros cuentos. Asunción: Araverá, 1984

WILLIAMS, John Hoyt. Esclavos y pobladores: observaciones sobre la historia parda del Paraguay en el siglo XIX. En Pasado y presente de la realidad social paraguaya. Vol. 1, Historia Social, Asunción: CPES, p. 685-705, 1995.

WALKER, Alice. In Search of our Mother's Gardens: Womanist Prose. San Diego, CA. Harcourd Brace, 1983.

WESCHENFELDER , V. I.; SILVA, M. L. A cor da mestiçagem: o pardo e a produção de subjetividades negras. Análise Social, v. 2, n. 227, Lisboa, 2018. p. 308-330. 


\section{Adjunto. Datos de filiación de pardos mencionados}

Nombre: Marcelino Godoy

Señas:

Natural de: Capiatá

Edad: 30 años

Estatura: 7 cuartas $(1,60 \mathrm{~m})$

Color: trigueño

Pelo: crespo

Cejas: grandes

Ojos: grandes

Cara: redonda

Nariz: corta y puntiaguda

Boca: grande, buena dentadura, labios gruesos

Barba: poca

Cuerpo: retacón

Vestimenta:

Gorra: tasa negro y casco punzó

Camiseta: de agujeta rosada con cuello y puños azules

Pantalón: de lienzo americano

Nombre: Manuel Otazú

Señas:

Natural de: Capital

Estatura: alta

Cuerpo: delgado

Color: pardo

Pelo: crespo

Barba: ralo

Vestimenta:

Pantalón: roto de casimir café

Camisa: de hilo del coco

Poncho: criollo azul viejo

Sombrero: de palma sucio

Nombre: Francisco Toledo

Señas:

Natural de: Santa María, Misiones

Estado: soltero

Edad: 29 años

Estatura: 8 cuartas $(1,82 \mathrm{~m})$

Color: tostado

Cuerpo: robusto

Cara: redonda

Cabeza: chica

Pelo: negro y crespo

Cara: redonda, picado por viruela

Cejas: poco poblado

Ojos: en color sanguinolento

Nariz: delgada

Boca: regular con buena dentadura

Barba: lampiño, sin bigote

Vestimenta:

Gorra: vieja de Infantería

Camiseta: rota con bayeta colorada

Pantalón: viejo sucio de lienzo americano
Nombre: Fermín Acosta

Señas:

Natural de: Barrero Grande

Altura: alto

Cuerpo: algo fornido

Color: tostado

Pelo: liso y grueso

Barba: sin pelo

Vestimenta:

Pantalón: chiripa blanco de lana

Camisa: de hilo del coco

Poncho: caris viejo

Sombrero: de palma sucio

Nombre: Francisco Vera

Señas:

Natural de: Villarrica

Edad: 31 años

Estatura: 5 pies $(1,52 \mathrm{~m})$

Color: aindiado

Cuerpo: corpulento

Pelo: negro y liso

Cara: redonda

Ojos: chico y negro

Nariz: corta

Boca: regular con buena dentadura

Bigote: raso, sin barba

Vestimenta:

Gorra: de Infantería de baja punzó

Camiseta: usada con bayeta colorada, con cuello y puños azules

Camisa: de bramante de regular uso

Pantalón: lienzo asargado

Poncho: negro bastante roto de paño azul en la estrella 
Nombre: Lázaro Barreto

$\underline{\text { Señas }}$

Natural de: Limpio

Estado: soltero

Edad: 40 años

Estatura: 7 cuartas $(1,60 \mathrm{~m})$

Color: moreno

Cuerpo: robusto

Cabeza: grande

Pelo: lizo

Cara: redonda

Ojos: negros

Cejas: poco poblada

Nariz: chata

Boca: grande con buena dentadura

Bigote: bien poblado

Vestimenta:

Lleva las insignias militares rotas y sucias
Nombre: Dionisio Escurra

Señas:

Natural de: Villarrica

Edad: 41 años

Estatura: 5 pies $(1,52 \mathrm{~m})$

Color: aindiado

Cuerpo: robusto

Pelo: negro y liso

Cara: redonda

Cejas: delgada

Nariz: corta

Boca: grande sin dientes

Vestimenta:

Gorra: vieja en faja punzó

Camiseta: vieja de bayeta colorada a mangas rotas y sin camisa

Pantalón: roto y sucio de lienzo asargado

Poncho: viejo de color grana

Recebido em 09/10/2021

Aprovado em 13/12/2021 